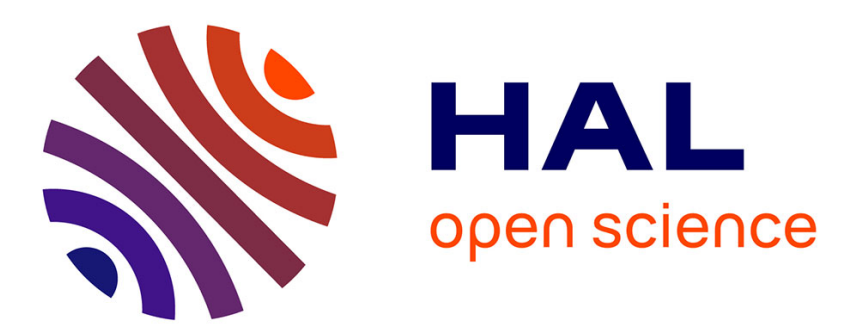

\title{
Analyse numérique de la méthode des variables adiabatiques pour l'approximation de l'hamiltonien nucléaire.
}

Yvon Maday, Gabriel Turinici

\section{- To cite this version:}

Yvon Maday, Gabriel Turinici. Analyse numérique de la méthode des variables adiabatiques pour l'approximation de l'hamiltonien nucléaire.. Comptes rendus de l'Académie des sciences. Série I, Mathématique, 1998, 3, pp.397-402. hal-00536513

\section{HAL Id: hal-00536513 https://hal.science/hal-00536513}

Submitted on 8 Mar 2013

HAL is a multi-disciplinary open access archive for the deposit and dissemination of scientific research documents, whether they are published or not. The documents may come from teaching and research institutions in France or abroad, or from public or private research centers.
L'archive ouverte pluridisciplinaire HAL, est destinée au dépôt et à la diffusion de documents scientifiques de niveau recherche, publiés ou non, émanant des établissements d'enseignement et de recherche français ou étrangers, des laboratoires publics ou privés. 
Analyse numérique/Numerical analysis

\title{
Analyse numérique de la méthode des variables adiabatiques pour l'approximation de l'hamiltonien nucléaire
}

\author{
par Yvon Maday et Gabriel Turinici
}

Résumé - De nombreux problèmes en chimie quantique portent sur le calcul d'états fondamentaux ou excités de molécules et conduisent à la résolution de problèmes aux valeurs propres. Une des difficultés majeures dans ces calculs est la très grande dimension des systèmes qui sont en présence lors des simulations numériques. En effet les modes propres recherchés sont fonctions de $3 n$ variables ou $n$ est le nombre de particules (électrons ou protons) de la molécule. Afin de réduire la dimension des systèmes à résoudre les chimistes fourmillent d'idées intéressantes qui permettent d'approcher le système complet. La méthode des variables adiabatiques entre dans ce cadre et nous présentons ici une étude mathématique rigoureuse de cette approximation. En particulier nous proposons un estimateur a posteriori qui pourrait permettre de vérifier l'hypothèse d'adiabaticité fait sur certaines variables.

\section{Numerical Analysis of the Adiabatic Variable Method for the Approximation of the Nuclear Hamiltonian.}

Abstract - Many problems in quantum chemistry deal with the computation of fundamental or excited states of molecules and lead to the resolution of eigenvalue problems. One of the major difficulties in these computations lies in the very large dimension of the systems to be solved. Indeed these eigenfunctions depend on $3 n$ variables where $n$ stands for the number of particles (electrons or protons) in the molecule. In order to diminish the size of the systems to be solved, the chemists have proposed many interesting ideas. Among those stands the adiabatic variable method; we present in this Note a mathematical analysis of this approximation and propose, in particular, an a posteriori estimate that might allow for verifying the adiabaticity hypothesis that is done on some variables.

\section{Abridged English Version-}

One problem frequently encountered in computational chemistry consists in the evaluation of the eigenmodes of some Hamiltonian operator corresponding to eigenvalues smaller than some prescribed value $E_{M A X}$. Even when only the nuclear Hamiltonian, under the Born-Oppenheimer approximation, is considered, the size of the molecule induces sizes of computations that rapidly grow huge. In order to diminish it, it is important to consider suitable discrete spaces and suitables basis functions of these discrete space. Any empirical argument leading to the definition of adapted basis and spaces is wellcomed in this direction. The pseudo-spectral adiabatic variable method proposed in [5], [6] is one pertinent discretization tool that seems to give quite good results in practice. We consider problem (1), (2) set on the cube $\Omega=\left[-1,1\left[^{2} \times\right] 0, \pi\left[\right.\right.$ of $\mathbb{R}^{3}$ that models the behaviour of a molecule composed of 3 atoms. In this case the method consists in first defining the space $X_{M, N}$ spanned by the eigenfunctions $\varphi_{k, \ell, n}(R, r, z)=\sin \left(\frac{k \pi}{2}(R+1)\right) \sin \left(\frac{\ell \pi}{2}(r+1)\right) L_{n}(z)$ of the operator $T_{R, r, z}$ defined in (3), with $1 \leq k, \ell \leq M, 0 \leq n \leq N$; we then diagonalize in $X_{M ; 0}$ the $N+1$ reduced (two dimensional) Hamiltonians $H^{r}\left(z=\xi_{i}\right)$ in (3), where the $\xi_{i}$ are the $N+1$ Gauss-Legendre quadrature points. We denote by $\left(\Phi_{p, q, i}(R, r), \Lambda_{p, q, i}\right)$ these eigenmodes and introduce the final discretization space $\mathcal{E}_{\delta}$ spanned by those elements $\Phi_{p, q, i}(R, r) h_{i}(z)$ with eigenmodes $\Lambda_{p, q, i} \leq(1+\varepsilon) E_{M A X}$. Here the polynomials $h_{i}$ are the characteristic functions that satisfy $h_{i}\left(\xi_{j}\right)=\delta_{i, j}$. The method consists now in diagonalizing $H_{\delta}$ defined in (4) over $\mathcal{E}_{\delta}$.

We present in this note an a priori error analysis that proves first that this method is converging but more importantly may provide some hint on the proper choice of the adiabatic variable (which in our case is $z=\cos (\theta)$ ). This error bound reads (8) whenever $\psi_{0} \in X_{0}^{s}$ and $V \psi_{0} \in L^{2}(]-$ $1,1\left[^{2} ; H^{t}(]-1,1[)\right) \cap H^{\tau}(]-1,1\left[^{2} ; L^{2}(]-1,1[)\right)$. Here $X_{0}^{s}$ is the domain of the operator $T_{R, r, z}^{s / 2}$ with homogeneous Dirichlet boundary conditions.

Then comes the a posteriori error bound that allows to check the validity of the adiabaticity reduction hypothesis. The analysis follows the general method introduced in [4] and leads to the (two sided) estimator 
Theorem 2. There exists constants $c$ and $C$ such that (11) holds together with (12)

In these estimates, $\pi_{\mathcal{E}_{\delta}}$ is the $L^{2}$ projection operator over the reduced space $\mathcal{E}_{\delta}$.

1. Introduction. - Un des problèmes fréquemment rencontrés dans les calculs scientifiques en chimie quantique (cf. [5] - [10]) est la recherche des valeurs/fonctions propres de l'hamiltonien nucléaire ayant une énergie plus petite qu'une valeur $E_{M A X}$ fixée à l'avance. L'hypothèse de Born-Oppenheimer amène à un hamilonien nucléaire ayant la forme $H=T+V$ où $V$ désigne un potentiel supposé connu dans les cas qui nous intéressent (soit empiriquement soit par calcul électronique préalable) et $T$ est l'opérateur cinétique de Laplace écrit dans divers systèmes de coordonnées.

Ce problème est posé en dimension de variables importante et tout argument tendant à améliorer les méthodes d'approximation numérique permet de repousser les limites du calcul. En particulier, il semble naturel d'introduire les premiers modes propres de l'opérateur de Laplace dans le système de coordonnées correspondant et chercher les modes propres de l'hamiltonien dans cette base modale. Pour ce faire on utilise une méthode itérative du type Lanczos qui repose sur le calcul d'une suite de vecteurs $\left\{\psi_{n}\right\}_{n}$ définis de manière récurente par $\psi_{n+1}=c_{0} H\left(\psi_{n}\right)-c_{1} \psi_{n-1}$.

Du point de vue de l'efficacité la partie la plus coûteuse est le calcul de $H\left(\psi_{n}\right)$. En effet, même si la base choisie est bien adaptée pour le Laplacien (puisqu'il est alors diagonal), la matrice représentant le potentiel $V$ est pleine.

Comme en général on s'intéresse à un spectre assez large, la taille de la base (et donc de la matrice en question) est importante à un point tel qu'il interdit souvent tout calcul. On est alors amené à chercher des méthodes pour réduire encore le nombre de fonctions de base. La réduction adiabatique (pseudo-)spectrale est l'un de ces procédés, largement utilisé dans la pratique, cf [5] et [6]. Son principe est exposé ci après sur le cas d'une molécule triatomique.

L'opérateur de Laplace est exprimé en coordonnées Jacobi $(R, r, \theta)$. On se propose ainsi de résoudre sur le cube $\Omega=\left[-1,1\left[{ }^{2} \times\right] 0, \pi\left[\right.\right.$ de $\mathbb{R}^{3}$ :

$$
\begin{aligned}
& \tilde{H} \psi=E \psi, \quad \text { où } \tilde{H}=\tilde{T}_{R, r, \theta}+V=-\partial_{R R}-\partial_{r r}-\frac{f(R, r)}{\sin \theta} \partial_{\theta} \sin \theta \partial_{\theta}+V \\
& \psi( \pm 1, r, \theta)=\psi(R, \pm 1, \theta)=0
\end{aligned}
$$

Alors

1 On identifie par une analyse en modes normaux autour de l'équilibre une variable spéciale pour le système qu'on va appeler la coordonnée adiabatique. Ici il s'agira par exemple de la variable $\theta$ et on écrit l'hamiltonien après changement de variable $z=\cos \theta$.

2 On considère l'hamiltonien obtenu en enlevant les termes contenant les dérivées dans la coordonnée adiabatique. Celui-ci sera appelé l'hamiltonien réduit, il s'agit ici de:

$$
H^{r}:=H+f(R, r) \partial_{z}\left(1-z^{2}\right) \partial_{z}=T_{R, r, z}+V+f(R, r) \partial_{z}\left(1-z^{2}\right) \partial_{z}=-\partial_{R R}-\partial_{r r}+V
$$

On le diagonalise par une procédure très rapide. En effet on ramène le problème $3 \mathrm{D}$ à un petit nombre de problèmes $2 \mathrm{D}$ en fixant la valeur de la coordonnée adiabatique. C'est ici qu'intervient l'intuition physique, la variable adiabatique étant d'une certaine façon celle qui permet de décrire au mieux l'hamiltonien total par son action en des valeurs fixées.

3 Puisqu'on cherche les vecteurs propres ayant une énergie plus petite que $E_{M A X}$, on ne garde parmi les vecteurs propres calculés à l'étape 2 que ceux dont l'énergie est plus petite que $(1+\epsilon) E_{\text {MAX }}$ (où $\left.\epsilon>0\right)$. 
4 En tensorisant les vecteurs obtenus au point 3 avec des fonctions caractéristiques de la variable adiabatique on définit une ensemble réduit de vecteurs où l'on cherche à diagonaliser $H$.

Dans la pratique le procédé donne de bons résultats; pourtant le choix de la (ou des) variable(s) adiabatique(s) et du système de coordonnées correspondant (cf. étape 1) influence beaucoup ses performances. Par conséquent il nous semble intéressant de donner des estimations a priori qui nous permettent d'avoir une certaine intuition du choix de la variable adiabatique pour un système donné et de compléter cette analyse numérique par des estimateurs a-posteriori afin d'en juger la pertinence une fois les calculs faits ainsi que de valider le choix de $\epsilon$ qui intervient dans la tronquature*.

Avant de procéder aux différentes analyses d'erreur, il est important de préciser le choix des valeurs de la variable adiabatique retenues qui vont être fixées au cours de l'étape 2 . Il s'agit du système des nœuds de la méthode d'intégration numérique de Gauss adaptée à la variable adiabatique. Ici l'opérateur est $\partial_{z}\left(1-z^{2}\right) \partial_{z}$ et son spectre est constitué des polynômes de Legendre $\left\{L_{n}\right\}_{n}$. Les valeurs fixées pour la variable adiabatique sont donc les points de Gauss-Legendre, il s'agit des racines $\left\{\zeta_{i}\right\}_{1 \leq i \leq N+1}$ du polynôme de Legendre $L_{N+1}$ de degré $N+1$. Il est classique d'associer à ces points la base des polynômes caractéristiques de degré $\leq N,\left\{h_{j}\right\}_{1 \leq j \leq N+1}$ tels que $h_{j}\left(\zeta_{i}\right)=\delta_{i, j}$ (symbole de Kronecker).

On introduit ici l'opérateur $\mathcal{J}_{N}$ d'interpolation de $\mathcal{C}^{0}(]-1,1[)$ dans $\mathbb{P}_{N}(]-1,1[)$.

2. Analyse A-Priori de la méthode - - Nous proposons cette analyse sur le cas particulier du système (1) et (2) à 3 particules avec $f(R, r) \equiv 1$. Comme on l'a vu, la discrétisation comporte deux étapes. On détermine tout d'abord les fonctions propres de l'opérateur $T_{R, r, z}$ sur $\left.L^{2}(]-1,1{ }^{3}\right)$, il s'agit des fonctions $\varphi_{k, \ell, n}(R, r, z)=\sin \left(\frac{k \pi}{2}(R+1)\right) \sin \left(\frac{\ell \pi}{2}(r+1)\right) L_{n}(z)$ pour $(k, \ell, n)$ parcourant $\mathbb{N}^{3}$. Puis on propose un premier espace de discrétisation $X_{M, N}$ engendré par les $\varphi_{k, \ell, n}$ pour $1 \leq k, \ell \leq M, 0 \leq n \leq N$. La seconde étape repose sur la détermination sur $X_{M, 0}$ des modes propres des opérateurs (de deux variables) $-\partial_{R R}-\partial_{r r}+V\left(., ., \zeta_{i}\right)$ pour chaque valeur de $i, 1 \leq i \leq N+1$, on appelle $\Phi_{p, q, i}$ et $\Lambda_{p, q, i}$ les vecteurs et les valeurs propres correspondants, on suppose ces premiers normalisés en norme $L^{2}$. L'approximation finale du problème consiste alors à chercher dans l'espace vectoriel $\mathcal{E}_{\delta}$ engendré par les $\Phi_{p, q, i}(R, r) h_{i}(z)$ (fonctions de 3 variables) correspondant aux valeurs propres $\Lambda_{p, q, i} \leq(1+\epsilon) E_{M a x}$ les fonctions propres de l'opérateur $H_{\delta}$ défini par

$$
\begin{gathered}
\left(H_{\delta} \varphi, \psi\right)=\int_{]-1,1\left[^{3}\right.} \partial_{R} \psi \partial_{R} \varphi+\partial_{r} \psi \partial_{r} \varphi+\left(1-z^{2}\right) \partial_{z} \psi \partial_{z} \varphi d R d r d z \\
+\int_{]-1,1\left[^{2}\right.} \sum_{i=1}^{N+1} V \psi \varphi\left(R, r, \zeta_{i}\right) \rho_{i} d R d r
\end{gathered}
$$

où les $\left\{\rho_{i}\right\}_{1<i<N+1}$ sont les poids de la formule de Gauss Legendre.

Remarque: Il est intéressant de noter que les $\Phi_{p, q, i}(R, r) h_{i}(z)$ sont les fonctions propres sur $X_{M, N}$ de l'opérateur $H_{\delta}^{r}$ défini comme suit

$$
\left(H_{\delta}^{r} \varphi, \psi\right)=\int_{]-1,1\left[^{2}\right.} \sum_{i=1}^{N+1}\left(\left(\partial_{R} \psi \partial_{R} \varphi+\partial_{r} \psi \partial_{r} \varphi\right)\left(R, r, \zeta_{i}\right)+V\left(R, r, \zeta_{i}\right)(\psi \varphi)\left(R, r, \zeta_{i}\right)\right) \rho_{i} d R d r .
$$

* Cette méthode de "reduction adiabatique" présente quelques similarités avec la méthode de reduction de dimension utilisée en mécanique des structures. On refère à [11] pour une présentation de cette méthode ainsi que des estimateurs d'erreur adaptés. Néanmois la méthode et les techniques d'analyse sont differents. 
On définit d'abord des espaces associés à l'opérateur $T_{R, r, z}$ qui seront les analogues des espaces de Sobolev $H^{s} \cap H_{0}^{1}$; plus précisement on désigne par $X_{0}^{s}$ l'adhérence de $C_{0}^{1}(]-1,1\left[{ }^{3}\right) \cap C^{\infty}(]-1,1\left[^{3}\right)$ dans le domaine de $\left(T_{R, r, z}\right)^{s / 2}$ muni de sa norme canonique. Le Thm. 5.6 de [3] et le Thm. 2.3 de [1] tome 1 p.19 permettent de caractériser les $X_{0}^{s}$. On obtient par exemple:

$$
X_{0}^{2}=\left\{u \in H_{0}^{1} ; \partial_{R R} u, \partial_{r r} u, \partial_{R r} u, \sqrt{1-z^{2}} \partial_{R z} u, \sqrt{1-z^{2}} \partial_{r z} u,\left(1-z^{2}\right) \partial_{z z} u \in L^{2}(]-1,1\left[^{3}\right)\right\}
$$

On écrit ensuite le problème initial sous la forme abstraite: trouver $u \in L^{2} \times \mathbb{R}$ tel que $F(u)=0$, où $F$ est une application de classe $C^{1}$ entre $L^{2} \times \mathbb{R}$ et le dual $\left(X_{0}^{2}\right)^{*} \times \mathbb{R}$ de $X_{0}^{2} \times \mathbb{R}$. L'application $F$ est ici donnée par:

$$
\begin{aligned}
<F(\psi, \lambda),(\varphi, \mu) & >\left(X_{0}^{2}\right)^{*} \times \mathbb{R}, X_{0}^{2} \times \mathbb{R}^{2}=\int_{]-1,1\left[^{3}\right.}(H \varphi-\lambda \varphi) \psi+\mu\left(\int_{]_{-1,1\left[^{3}\right.}} \psi^{2}-1\right) \\
& =\int_{]-1,1\left[^{3}\right.}\left(T_{R, r, z} \varphi+V \varphi-\lambda \varphi\right) \psi+\mu\left(\int_{]-1,1\left[^{3}\right.} \psi^{2}-1\right)
\end{aligned}
$$

Il est facile à vérifier que $F\left(\psi_{0}, \lambda_{0}\right)=0$ est équivalent au problème (1)-(2). Par ailleurs si $\lambda_{0}$ est valeur propre simple de (1) correspondant au vecteur propre $\psi_{0}$ (normalisé dans $L^{2}$ ), alors par application de l'alternative de Fredholm on montre que $D F\left(\psi_{0}, \lambda_{0}\right)$ est un isomorphisme de $Z=L^{2} \times \mathbb{R}$ dans $Y=\left(X_{0}^{2}\right)^{*} \times \mathbb{R}$.

Soit ensuite $\Pi_{\delta}$ le projecteur sur $\mathcal{E}_{\delta}$ associé à $T_{R, r, z}$ i.e. pour tout $v \in X_{0}^{2}, \Pi_{\delta} v$ est l'element de $\mathcal{E}_{\delta}$ qui réalise $\int_{]_{-1,1}\left[^{3}\right.} T_{R, r, z}\left(v-\Pi_{\delta} v\right) u=0$ pour tout $u \in \mathcal{E}_{\delta}$. On définit alors des fonctions $F_{\delta}$ de $L^{2} \times \mathbb{R}$ dans $\left(X_{0}^{2}\right)^{*} \times \mathbb{R}$ par la formule:

$$
\begin{aligned}
<F_{\delta}(\psi, \lambda),(\varphi, \mu)>{ }_{\left(X_{0}^{2}\right)^{*} \times \mathbb{R}, X_{0}^{2} \times \mathbb{R}}=\int_{]-1,1\left[^{3}\right.}\left(H_{\delta}-\lambda\right)\left(\Pi_{\delta} \varphi\right) \psi \\
\quad+\mu\left(\int_{]_{-1,1\left[^{3}\right.}} \psi^{2}-1\right)+\int_{]-1,1\left[^{3}\right.} T_{R, r, z}\left(\varphi-\Pi_{\delta} \varphi\right) \psi
\end{aligned}
$$

Les fonctions propres de l'opérateur $H_{\delta}$ sur $\mathcal{E}_{\delta}$ sont alors exactement les solutions de $F_{\delta}\left(\psi_{\delta}, \lambda_{\delta}\right)=0$.

On applique ensuite le théorème 6.1 de [2] vol 5 p.530 qui permet de montrer que, sous certaines hypothèses, on a l'estimation a-priori désirée

$$
\begin{aligned}
& \left\|\psi_{0}-\psi_{\delta}\right\|_{L^{2}}+\left|\lambda_{0}-\lambda_{\delta}\right| \leq C(\tau, s, t)\left(\left(\epsilon_{\delta}\right)^{s}\left\|\left(\psi_{0}, \lambda_{0}\right)\right\|_{X_{0}^{s} \times \mathbb{R}}+\right. \\
& \left.N^{-t}\left\|V \psi_{0}\right\|_{L^{2}(]-1,1\left[^{2} ; H_{*}^{t}(]-1,1[)\right)}+M^{-\tau}\left\|V \psi_{0}\right\|_{H^{\tau}(]-1,1\left[\left[^{2} ; L^{2}(]-1,1[)\right)\right.}\right)
\end{aligned}
$$

où $\epsilon_{\delta}$ est $\sup \left\{\frac{1}{N}, \frac{1}{M}, \frac{1}{\sqrt{E_{\text {Max }}}}\right\}$ et $H_{*}^{t}(]-1,1[)$ est un espace à poids qui contient $H^{t}(]-1,1[$ ) (voir [3] pag. 24).

Remarque. Si $V$ est suffisemment régulière, il est clair que pour tout $p$ les normes $\left\|\psi_{0}\right\|_{X_{0}^{p}}$, $\left\|V \psi_{0}\right\|_{\left.L^{2}(]-1,1\left[^{2} ; H_{*}^{2 p}(]-1,1\right]\right)}$ et $\left\|V \psi_{0}\right\|_{H^{2 p}(]-1,1\left[^{2} ; L^{2}(]-1,1[)\right)}$ se comportent en $c\left|\lambda_{0}\right|^{p}$ ce qui fait que, pour le choix naturel $N^{2} \simeq M^{2} \simeq E_{\text {Max }}$ on a une convergence en $c(p)\left(\frac{\lambda_{0}}{N^{2}}\right)^{p}$.

3. Analyse a-posteriori de la méthode - - Toujours sur le cas particulier du système (1) et (2) à 3 particules, nous présentons maintenant une analyse de l'erreur a posteriori. Celle-ci 
a pour but de qualifier l'approximation une fois les calculs terminés. On travaille toujours dans la formulation $F(u)=0$.

L'estimation a priori montre que pour tout mode propre $\left(\psi_{0}, \lambda_{0}\right)$ simple du système (1)-(2), il existe un mode propre $\left(\psi_{\delta}, \lambda_{\delta}\right)$ qui lui est proche. La question de savoir de combien ils sont proches découle cette fois de résultats dérivés par exemple de [4] qui permettent de montrer que, sous certaines hypothèses, $F\left(\psi_{\delta}, \lambda_{\delta}\right)$ est un estimateur de l'erreur entre une solution exacte $\left(\psi_{0}, \lambda_{0}\right)$ et la solution approchée $\left(\psi_{\delta}, \lambda_{\delta}\right)$. Plus précisement on a:

$$
c\left\|F\left(\psi_{\delta}, \lambda_{\delta}\right)\right\|_{Y} \leq\left\|\psi_{0}-\psi_{\delta}\right\|_{L^{2}(]-1,1\left[^{3}\right)}+\left|\lambda_{0}-\lambda_{\delta}\right| \leq C\left\|F\left(\psi_{\delta}, \lambda_{\delta}\right)\right\|_{Y}
$$

pour deux constantes positives $c$ et $C$. On écrit dans une première étape

$$
\begin{aligned}
\left\|F\left(\psi_{\delta}, \lambda_{\delta}\right)\right\|_{Y} & =\sup _{\varphi \in X_{0}^{2},\|\varphi\|_{X_{0}^{2}}=1} \int_{]-1,1\left[^{3}\right.}\left(\left(V \psi_{\delta}-\pi_{M} \mathcal{J}_{N}\left(V \psi_{\delta}\right)\right) \varphi\right. \\
& +\sup _{\varphi \in X_{0}^{2},\|\varphi\|_{X_{0}^{2}}=1} \int_{-1,1\left[^{3}\right.}\left(T_{R, r, z} \psi_{\delta}+\pi_{M} \mathcal{J}_{N}\left(V \psi_{\delta}\right)-\lambda_{\delta} \psi_{\delta}\right) \varphi_{M N}
\end{aligned}
$$

La première contribution du membre de droite mesure l'approximation due à la réduction de l'action de $V$ dans $X_{M N}$. La seconde contribution représente en revanche la perte d'information commise en négligeant dans $X_{M N}$ les modes $\Phi_{p, q, i} h_{i}$ d'énergie supérieure à $(1+\epsilon) E_{M a x}$. C'est cette contribution qui permet de juger de l'adiabaticité du système car il porte sur la projection du terme $\left(T_{R, r, z} \psi_{\delta}+\pi_{M} \mathcal{J}_{N}\left(V \psi_{\delta}\right)-\lambda_{\delta} \psi_{\delta}\right)$ sur les modes "oubliés". En effet sa projection sur les termes retenues est nulle, par définition de $\psi_{\delta}$. L'analyse précise de ces deux contributions permet d'obtenir:

Théorème 2. Il existe des constantes $c$ et $C$ telles que

$$
\begin{gathered}
\left\|\psi_{0}-\psi_{\delta}\right\|_{L^{2}(]-1,1\left[^{3}\right)}+\left|\lambda_{0}-\lambda_{\delta}\right| \leq \frac{c(V)}{E_{M a x}}\left\|\left(I d-\pi_{\mathcal{E}_{\delta}}\right)\left(\partial_{z}\left(1-z^{2}\right) \partial_{z} \psi_{\delta}\right)\right\|_{L^{2}(]-1,1\left[^{3}\right)} \\
+c\left(N^{-t}\left\|V \psi_{\delta}\right\|_{L^{2}(]-1,1\left[^{2} ; H_{*}^{t}(]-1,1[)\right.}+M^{-\tau}\left\|V \psi_{\delta}\right\|_{H^{\tau}(]-1,1\left[^{2} ; L^{2}(]-1,1[)\right)}\right)
\end{gathered}
$$

et

$$
\begin{gathered}
\frac{c}{\sup (M, N)^{2}}\left\|\left(I d-\pi_{\mathcal{E}_{\delta}}\right)\left(\partial_{z}\left(1-z^{2}\right) \partial_{z} \psi_{\delta}\right)\right\|_{L^{2}(]-1,1\left[^{3}\right)} \leq\left(\left\|\psi_{0}-\psi_{\delta}\right\|_{L^{2}(]-1,1\left[^{3}\right)}+\left|\lambda_{0}-\lambda_{\delta}\right|\right) \\
+c\left(N^{-t}\left\|V \psi_{\delta}\right\|_{L^{2}(]-1,1\left[^{2} ; H_{*}^{t}(]-1,1[)\right.}+M^{-\tau}\left\|V \psi_{\delta}\right\|_{H^{\tau}(]-1,1\left[^{2} ; L^{2}(]-1,1[)\right.}\right)
\end{gathered}
$$

Remarque 1. D'après l'estimation a priori (et le bon sens) il est naturel de choisir $N^{2} \simeq M^{2} \simeq$ $E_{\text {Max }}$. Le théorème 2 donne donc une estimation a posteriori optimale pour juger de l'adiabaticité de la variable.

Remarque 2. Le calcul explicite de la contribution:

$$
\left\|\left(I d-\pi_{\mathcal{E}_{\delta}}\right)\left(\partial_{z}\left(1-z^{2}\right) \partial_{z} \psi_{\delta}\right)\right\|_{L^{2}(]-1,1\left[^{3}\right)}
$$

peut se faire rapidement de la façon suivante: on écrit tout d'abord

$$
\Phi_{p, q, i}(R, r)\left(\partial_{z}\left(1-z^{2}\right) \partial_{z} h_{i}\right)(z)=\sum_{p^{\prime}, q^{\prime}=1}^{M} \sum_{j=1}^{N} \alpha_{p q i}^{p^{\prime} q^{\prime} j} \Phi_{p^{\prime}, q^{\prime}, j}(R, r) h_{j}(z)
$$


Partant de l'écriture $\psi_{\delta}=\sum_{(p, q, i) ;\left|\Lambda_{p, q, i}\right| \leq(1+\epsilon) E_{M a x}} \check{\psi}_{p q i} \Phi_{p, q, i} h_{i}$ donnée par la résolution du problème réduit on en déduit:

$$
A \psi_{\delta}:=\left[\partial_{z}\left(1-z^{2}\right) \partial_{z}\right] \psi_{\delta}=\sum_{p^{\prime}, q^{\prime}, j}\left[\sum_{(p, q, i) ;\left|\Lambda_{p, q, i}\right| \leq(1+\epsilon) E_{M a x}} \alpha_{p q i}^{p^{\prime} q^{\prime} j} \check{\psi}_{p q i}\right] \Phi_{p^{\prime}, q^{\prime}, j}(R, r) h_{j}(z)
$$

Ceci fournit la valeur des coefficients de $A \psi_{\delta}$ dans la base orthonormée $\Phi_{p^{\prime}, q^{\prime}, j}(R, r) h_{j}(z)$ et donc la norme $L^{2}$ de $\left(I d-\pi_{\mathcal{E}_{\delta}}\right) A \psi_{\delta}$ en découle. Par tensorisation le calcul (13) peut se faire en $c \max \{M, N\}^{5}$ opérations, moins que le nombre d'opérations demandé pour le calcul de $\psi_{\delta}$.

Remerciements Les auteurs tiennent à remercier C. Leforestier du Laboratoire Structure et Dynamique des Systèmes Moléculaires et Solides de l'Université de Montpellier 2 pour les discussions sur le sujet.

\section{REFERENCES}

[1] J.L.Lions et E.Magenes "Problèmes aux limites non-homogènes et applications", DUNOD, Paris 1968

[2] "Handbook of numerical analysis" (Part 2) ed. par Ciarlet, Philippe G. et Lions, Jacques Louis London ; New York NY ; Tokyo : North-Holland, 1991

[3] M. Azaiez, M. Dauge et Y. Maday "Méthodes spectrales et les éléments spectraux" Institut de Recherche Mathématique de Rennes

[4] R.Verfürth "A Review of A Posteriori Error Estimates and Adaptative Mesh-Refinement Techniques" Wiley-Teubner 1997

[5] R.Friesner, J.Bentley, M.Menou and C.Leforestier, "Adiabatic pseudospectral methods for multimensional vibrational potentials" J.Chem.Phys., 99 , 324(1993).

[6] C.Leforestier "Grid representation of rotating triatomics", J.Chem.Phys., 94 , 6388(1991).

[7] K.Yamashita, K.Mokoruma and C.Leforestier "Theoretical study of the highly vibrationally excited states of $\mathrm{FHF}^{-}$: Ab initio potential energy surface and hyperspherical formulation", J.Chem.Phys., 99 , 8848(1993).

[8] J.Antihainen, R.Friesner and C.Leforestier "Adiabatic pseudospectral calculation of the vibrational states of the four atom molecules: Application to hydrogen peroxide" J.Chem.Phys., $102,1270(1995)$.

[9] D. Kosloff et R.Kosloff "Fourier Method for the Time Dependent Schrödinger Equation as a Tool in Molecular Dynamics" J. Comp. Phys. vol 52, 35 (1983)

[10] R.Kosloff "Time-Dependent Quantum-Mecanical Methods for Molecular Dynamics" J. Chem. Phys., 92, 2087(1988).

[11] I. Babuška and C. Schwab "A posteriori error estimation for hierarchic models of elliptic boundary value problems on thin domains" SIAM J. Numer. Anal. Vol 33 (1996), No.1, pp 241-246 